\title{
Presupuestos filosóficos del pecado original en San Agustín
}

En una Semana de teología sobre el pecado original el nombre de san Agustín no podía estar ausente, o quizá debiera estarlo para dejar libertad al tratado, ya que ha impuesto ciertas categorías al tema, de las que la teología posterior se ha desligado muy difícilmente. San Agustín ha copado casi toda la doctrina sobre el pecado original y el mismo magisterio ha bebido en su fuente. Su autoridad ha pesado a veces con exceso y hoy se ha lamentado con frecuencia la fuerza impresionante de su palabra. Situarlo en su contexto histórico sería un primer paso para no garantizarle una autoridad infalible; estudiarlo como mera historia del pensamiento sería concederle muy poco, como enmarcarlo en el cuadro de la tradición, nos haría ver su nueva aportación o profundización en el tema. Lo cierto es que, por exagerarse de una parte su autoridad, se le ha desvirtuado por otra, haciéndole responsable de la invención del pecado original ${ }^{1}$. En la historia de la teología ese mote ha sonado repetidamente, pero se ha extendido mucho más en nuestros días y se ha llevado hasta ei extremo en algunos estudios, que han obligado otros para una puesta en punto ${ }^{2}$.

LOS ESTUDIOS REALIZADOS.

Será difícil decir algo nuevo en torno al pecado original y su problemática en san Agustín, si nos atenemos a la inmensa bibliografía sobre ello ${ }^{3}$. Se ha estudiado principalmente su doctrina en la polémica pela-

1 Cfr. Turmex, J., "Lo dngrme du péché originel dans saint Augustin": $R e$ Entstehungsgeschichte Littérature Kercyiu... G (1901) 385-426, 404; Gross, J.,

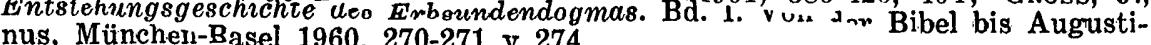

2 Veremos luezo la primera controversia sobre el tema.

3 Cfr. BuonaIUTI, E., La genesi della dottrina agostiniana intorno al peccato originale, Roma 1916; TURMEL, J., "Le dnome du péché originel avant saint 
giana, examinando las pruebas de la existencia del mismo, en el bautismo de los infantes, en la universalidad de la redención, en las miserias del hombre y de la creación entera ${ }^{4}$. Unido a esto y con idéntica visión se ha intentado resolver la espinosa cuestión de la esencia y de la naturaleza del pecado original ${ }^{5}$, contando con la diversidad de interpretaciones en el decurso de la historia de la teología ${ }^{6}$, llegando a confundirse

Augustin": Revue d'Histoire et de Littérature Religieuse 5 (1900) 503-526; 6 (1901) 13-31; "Le dogme du péché originel dans saint Augustin". Ib. 6 (1901) 235-258, 385-426; 7 (1902) 128-146, 209-230, y luego publicados en Histoires des dogmes, I, Paris 1931; Gross, J., o. c.; ANRUP, N. E., Augustinus lära om arvysnden. En dogmhistorisk studie. (Doctrina sancti Augustini de peccato originali), Lund 1943; MERLIN, N., Saint Augustin et les dogmes du péché originel et de la grace. Analyses detaillées de ses ouvrages sur ces matieres, Paris 1931; Pincherle, A., La formazione della docttrina agostiniana del peccato originale. (Estratto delle Annali della Facoltá di lettere e filosofia della R. Universita di Cagliari, IX, 5). Cagliari 1939; LEgEWIE, Sanktus Aurelius Augustinus. Uber die Erbsünde. Herausgegeben von... (Sexualpsychologie, 3. Dokumente zur Geschichte der Sexualität, 3), Lörrach (Baden), 1928; Williams, N. P., The Ideas of the Falls and of original Sin, 2 ed., London 1929; Willis, J. R., The Teaching of the Church Fathers, New York 1966, recoge unos textos en p. 265-78; RONDET, H., Le péché originel dans la tradition patristique et théologique, Paris 1967, sobre san Agustín y la tradición agustiniana, p. 134-173. Podríamos a su vez enumerar también los artículos generales en los Diccionarios o Enciclopedias, pero nos creemos dispensados de hacerlo, al igual que las referencias al tema en otros escritos sobre la doctrina del Santo.

4 Cfr. además de los citados en la nota precedente Philips, G., "De argumento agustiniano ex miseriis hujus vitae desumpto pro existentia peccati originalis": Revue Ecclésiastique de Liege 26 (1934-1935') 313-320.

5 Cfr. Dubarle, A. M., "Bulletin de théologie: Péché originel: Recherches recentes et orientations nouvelles": Revue de Sciences Philosophiques et Théologiques 53 (1969), 81-113, con un apartado, el 7. La problématique augustinienne du péché originel.

6 DE BLIC, J., "Le péché originel selon saint Augustin": Recherches de Science Religieuse 16 (1926) 97-119; 17 (1927) 414-433, 512-531. Una ojeada sobre la interpretación de los textos agustinianos relativos al pecado original es su primer intento. $Y$ divide las interpretaciones en tres series sucesivas: anteriores a la Reforma, después de la Reforma y las de los últimos años. Puede decirse -escribe - de una manera general que todo el siglo XII ha visto en san Agustín el pecado original definido por la concupiscencia (p. 97). En el segundo artículo habla de dos sistemas de interpretación que es preciso descartar. Primero el sistema de la privación de la gracia santificante, en el que examina la semejanza de Dios, ser solamente criatura, o sea simples animales racionales, no hijos de Dios; y segundo el sistema de la solidaridad moral con Adán. Su resumen podría ser éste, con las tres tendencias de interpretación. La primera, en fecha, la que identifica pecado original y concupiscencia, trata de hacer unanimidad en un tiempo en que el pensamiento de Agustín reina solamente en las escuelas y se impone a un estudio exclusivo. Cuando el favor de las fórmulas anselmianas y la necesidad de conciliar dos doctores de la Iglesia obligan a un cierto número de escolásticos a explicar a Agustín por Anselmo -con una intención más dialéctica que positiva-, otros por hacer nrofesión de puro agustinis.

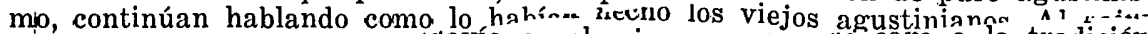
cipio del siglo XVI so cu coctavía en el mismo punto: ae cara a la tradición atiofine el pecado original por la concupiscencia, es sinple afirmourun desprovista de pruebas y lanzada de paso, que no se trata allí más que de lo material del pecado. Es preciso que intervenga la Reforma y la alternativa en que se encuentran los controversistas do combatir netamente el agustinismo 
el pecado originail con la concupiscencia ${ }^{7}$. En relación con la concupiscencia y con el origen del alma se ha hablado de la transmisión del pecado por generación, dando esto como un hecho ${ }^{8}$, recogido en expresión al menos por los documentos de la Iglesia ${ }^{9}$.

Además de esto, se le ha achacado de nuevo a Agustín el maniqueismo, en la concienciá de que ha sustituído el principio del mal por el pecado originai, como hicieran ya los pelagianos, de modo especial Julián de Eclana ${ }^{10}$. Y ese dualismo se ha aplicado a toda la doctrina agustiniana, con referencia primordial a la doctrina del matrimonio ${ }^{11}$, en la que no se vería libre, según esta interpretación, de un cierto dualismo y de la malicia de la carne, que viciaría sus profundas intuiciones. Se ha estu-

tradicional, o de admitir un acuerdo parcial entre Agustín y Lutero, para que tomen cuerpo no una, sino otras dos explicaciones: la de la participación de la humanidad en la falta personal de Adán, y la de la privación de la gracia santificante -esta última la más escolástica de las dos-, debiendo esperar hasta el siglo XIX los textos necesarios para su autentificación" (p. 414-415). DE BLIC; no pudo concluir su artículo debido a su muerte y continuará Clemence, J., "Saint Augustin et le péché originel": Nouvelle Revue Théologique 70 (1948) 727-754; cfr. también ALSZEGHY, Z., "La teologia dell'ordine soprannaturale nella scolastica antica": Gregorianum 31 (1950) 414-450, con una buena bibliografía, y LotTin, O., "Le péché originel chez Albert le Grand, Bonaventure et Thomas d'Aquin": Recherches de Théologie Ancienne et Médiévale 12 (1940) 275-328; "Bapteme ot péché originel de S. Anselme a S. Thomas d'Aquin": Ephemerides Theologicae Lovanienses 19 (1942) 225-245.

7 DAVILA, C., La concupiscencia y su relación con el pecado original y originado, Bogotá 1947; ORBE, R., "San Agustín y el problema de la concupiscencia en su marco histórico": Revista Española de Teología 1 (1940-1941) 313-337. 8 GibelliNi, R., La generazione come mezzo di trasmissione del peccato originale, Brescia 1965, citando en la p. 10 los artículos que ha publicado a este respecto; cfr. DE LETTER, P., "The transmission of original Sin": The Irish Theological Quarterly 24 (1957) 339-345; LONCKE, J., "De peceati originalis in genus humanum transmissione": Collaciones Brugenses 44 (1948) 413-417; Romuado De Palma, "Les causes de la tranmissió del pecat original": Estudis Franciscans 39 (1927) 210-219.

${ }_{9}$ Cfr. KocH, W., "Das Trienter Konzilsdekret de peccato originali": Theologische Quartalschrift 95 (1913) 430-450, 532-564; 96 (1914) 101-123; CAVALLERA, F., "Le décret du Concile de Trente sur le péché originel": Bulletin de Littérature Ecclésiastique 4 (1913) 241-258, 289-315; VANNESTe, A., "La Préhistoire du décret du Concile de Trente sur le péché originel”: Nouvelle Revue Theologique 86 (1964) 335-368, 490-510; "Le Décret du Concile de Trente sur le péché original": Ibid. 87 (1965) 688-721.

10 Cfr. Op. imp. contra Julian. IV, 1, PL 44, 1.338; I, 97, col. 1.113; II, $20-22$, cols. $1.149-1.150$; 32-33, col. 1.155 ; 117 , col. 1.191 y en otros muchos lugares, en que está en juego la aplicación de maniqueos a los católicos y en especial a Agustín mismo.

11 Cfr. JANssens, L., "Morale conjugale et progéstogenes": Ephemerides Theologicae Lovaienses 39 (1963) 787-826; MoRAN, J., "Sexualidad, humanización y pecado original": Archivo Teológico Agustiniano 1 (1966) 215-244; Janssens, L., Mariage et fecondite. De "Casti Connubii" a "Gaudium et Spes". Gombloux-Paris 1967, 13-41, La doctrine augustinienne; NoonaN, J. T., Contraception et Mariage. Evolution ou contradiction dans la pensée chrétienne?, Paris 1969, 141-182, y luego 221-256. En la mayoría de los estudios sobre el matrimonio se tiende hoy a esta interpretación. 
diado también en él la relación con los Concilios de Mỉievi (a. 4r6), con el Concilio de Cartago del $4{ }^{1} 8^{12}$, y su influjo en el Concilio de Orange ${ }^{13}$ y en la formulación del Concilio de Trento ${ }^{14}$, que recogería las fórmulas de aquél con las nuevas interpretaciones. A veces se ha buscado también en éi lo dogmático, siguiendo la tradición de la iglesia antigua y dejando libertad en lo discutible, siendo sus propuestas meramente personales, aunque hayan pesado en la historia. En este sentido sería fácilmente comprensible y tratando de extraer lo dogmático, sus opiniones personales no tendrían más valor que las de cualquiera otro autor privado con las perspectivas y la ciencia de su tiempo ${ }^{15}$. Se ha pensado también en la evolución de su pensamiento a este respecto ${ }^{16}$ y se ha tratado de ver el influjo de ciertos textos de la Escritura y la interpretación de los mismos por él y por algunos autores antiguos ${ }^{17}$. La evolución en ciertos puntos no podría ponerse en duda, si bien quizá no en aquellos en que algunos autores pensaban ${ }^{18}$.

A su vez y con buen criterio se ha estudiado la terminología, aunque

12 BONNER, G., "Les origines africaines de la doctrine augustinienne sur la chute et le péché originel": Augustinus 12 (1967) 97-116; FLORRI, F., "Le pape Zozime et la doctrine augustinienne du péché originel": Augustinus Magister II, 755-761, Paris 1954, y la discusión III, 261-263, Paris 1955; "Remarques sur la doctrine augustinienne du péché originel": Studia Patristica IX (Texte und Untersunchungen 94), 416-421, Berlin 1966; REFoulE, F. R., Datation du premier Concile de Quarthage contre les Pélagiens et du Libellus fidei de Rufin": Revue des Etudes Augstiniennes 9 (1963) 41-49. Vid. ambién A. SAGE.

18 Cfr. CappuYNs, M., "L'origine des "capitula" d'Orange 529": Recherches de Théologie Ancienne et Médiévale 6 (1934) 121-142; FrITz, G., "Orange" (deuxieme Concile d'): Dictionnaire de Théologie Catholique XI (1931) col 1.0871.103 .

14 Cfr. la nota 9, y Penagos, L., "La doctrina del pecado original en el Concilio de Trento": Miscellanea Comillensis 4 (1945) 127-273; GuTwENGER, E., "Die Erbsünde und das Konzil von Trient": Zeitschrift fur Katholische Teologie 89 (1967) 433-446.

15 SACE, A., "Le péché originel dans la pensée de saint Augustin, de 412 a 430": Revue des Etudes Augustiniennes 15 (1969) 75-112.

16 Sage, A., "Péché originel. Naissance d'un dogme": Revue des Etudes Augustiniennes 13 (1967) 211-248. Y en él se hacen otras afirmaciones por el estilo, citando a autores diversos.

17 Cfr. Lyonne, S., “Le sens de Eph'o en Rom. 5, 12 et l'exegese des Peres Grecs": Biblica 36 (1955) 436-456; "Le péché originel en Rom. 5, 12. L'exegese des Peres grees et les décrets du concile de Trente": Biblica 41 (1960) 325-355; Romani 5, 12 in Sant'Agostino. Nota sull elaborazione della dottrina agostiniana del peccato originale. L'uomo davanti a Dio, Alba-Roma 1966, 393-413; MEHLMANN, J., Natura filii irae. Historia interpretationis Eph. 2, 3 eiusque cum doctrina de peccao originali nexus, Roma 1957. Y en casi todos los estudios se examinan algunos textos agustinianos en referencia con la Escritura.

18 Cfr. Casamassa, A., Il pensiero di sant'Agostino nel 396-397. I "Tractatores divinorum eloquiorum" di Rteract. I, 23, le l'Ambrosiastro, Roma 1919, publicado ahora en Scritti patristici I. (Lateranum n. s., an. XXI, n. 1-4, Roma 1955, 41-66). 
no de manera exhaustiva, y se ha distinguido el peccatum originale, el peccatum naturale y los peccata originalia, de que habla en alguna ocasión ${ }^{10}$ y se ha llegado a plantear la posibilidad de una "pluralidad de pecados hereditarios" 20. Quizá lo que no se haya estudiado todavía con profundidad sea el influjo de san Ambrosio a este respecto en su primera etapa y cómo interpreta Agustín los textos ambrosianos en la controversia pelagiana, lo cual podría abrir una pista a nuevos descubrimientos y enriquecimientos ${ }^{21}$, sin que descontemos tampoco el influjo del $\mathrm{Ambro-}$ siaster $^{22}$.

\section{NuESTRO inTENTO.}

Tras un examen de la doctrina bautismal en la controversia donatista, en la que se puso de relieve el in remissionem peccatorum y do circunstancial, aunque prolongado, de la polémica pelagiana, y además tras la lectura de la bibliografía, cuyo contenido hemos recogido brevemente en las líneas precedentes, me ha asaltado siempre la misma pregunta: ¿San Agustín ha llegado a concluir a la existencia dei pecado original, obligado por la tradición y la Escritura, o más bien por su propia experiencia personal, por su formación cristiana y sus concepciones filosóficas? ¿No habrán sido realmente la Escritura y la tradición un fuerte apoyo a su vivencia de pecado irresistible y a sus ideales filosóficos inasequibles? ¿Si no hubiera surgido el pelagianismo se hubiera expresado con tal acuidad sobre el pecado original, o hubiera más bien

19 Vid. nota 15.

20 Dubarle, A. M., "La pluralité des péchés héréditaires dans la tradition augustinienne": Revue des Etudes Augustiniennes 2 (1957) 113-136.

21 Cfr. Huhn, J., Ursprung und Wesen des Bösen und der Sünde nach der Lehre des Kirchenvaters Ambrosius. (Forschungen zur christlichen Literaturund Dogmengeschichte 17-5), Mainz 1933. El estudio comparativo lo tenemos en preparación.

22 Pueden verse a este respecto los artículos citados en las notas 31 y 32 , a lo que puede añadirse: Buonaluti, E., "Agostino e la colpa ereditaria": Ricerche religiose 2 (1926), 401, donde dice: "saint'Agostino ha conosciuto, verso il 395, i Tractatus dell'Ambrosiastro e vi ha attinto la metafora pregnante della massa peccati, da cui a sua volta ha ricavato, con un riferimento spontaneo alla massa di fango, da cui il ceramista ricava i vasi che vuole (Rom. IX, 21), corroborato anch'esso del resto del medesimi Tracatus, la concezione della insindacabile e imperscrutabile libertà di Dio nell'elezione dei santi"; ID., "Pelagio e L'Ambrosiastro": Ibid. 4 (1928) 11; LeEmIng, B., "Augustine, Ambrosiaster and the "massa perditionis": Gregorianum 11 (1930) 58-91. Estudia el problema con agudeza Pincherle, A., La formazione teologica di S. Agostino, Roma, s. f., 175-205 con las notas. 
continuado la línea tradicional que expresa en sus dos primeras etapas? He aquí el centro de nuestro estudio: Agustín concluye a una deficiencia inicial, porque piensa en el hombre ideal primero, en plena conciencia de valores de la que ahora no le queda más que un tenue recuerdo, un anhelo y una inquietud, y al pretender la unión con los valores y no poder conseguiria, se adhiere a una mancha original que le explica la imposibilidad del ideal filosófico y le obliga a la necesidad de Cristo.

Tendríamos dos puntos claves: su formación maniquea y su asentamiento platónico y plotiniano, prescindiendo por el momento de los influjos estoicos, que explicarían también algo a este respecto.

El maniqueismo con su mito del Anthropos le ha creado una conciencia. Aquel antrhopos inicial ha perdido los espíritus y tiene que venir nuevamente a recuperarlos para constituir su plenitud, su pléroma. Esa caída y esa pérdida vendrá en parte relacionada con el pecado original y el mito servirá más tarde a Agustín, cristianizando, para su construcción del Christus totus ${ }^{23}$. Hay un texto notabilísimo que quizá pueda hacer luz sobre la doctrina posterior. Es del De natura boni 46 (PL. 42, 569-570; a. 399), y en él se dice:

"Nam et a quibusdam principibus gentis tenebrarum sic dicunt Adam hominem creatum, ut lumen ab eis ne fugeret teneretur".

Y luego con texto de la Epistula Fundamenti explica todo el proceso, cómo han dado su luz al príncipe para poder conservarla y reinar. Lo han hecho por medio de la generación, y el príncipe al recibirlo todo con sus fuerzas y luces, se unió a su cónyuge, emanada de la misma estirpe,

"et facto cum ea coitu, seminavit, ut caeteri, abundantiam malorum quae devoraverat: nonnihil etiam ipse adjiciens ex sua cogitatione ac virtute, ut esset sensus ejus omnium eorum quae profuderat formator atque descriptor; cujus compar excipiebat haec, ut semen consuevit culta optime terra percipere. In eadem enim construebantur et contexebantur omnium imagines, coelestium ac terrenarum virtutum, ut pleni videlicet orbis, id quod formiabatur, similitudinem obtineret" 24.

El mito del anthropos tiene un valor inicial, al principio, y un vaior redentivo en ese que absorberá toda la malicia y dará la luz al mundo.

23 Cfr. Schenke, H. M., Der Gott "Mensch" in der Gnosis. Ein religionsgeschichtlicher Beitrag zur Diskussion über die paulinische Anschauung von der Kirche als Leib Christi, Gottingen 1962, 108-119 principalmente.

24 De natura boni 46, PL. 42, 570. 
Si Agustín se mantuvo durante tanto tiempo en el maniqueismo, aunque no estuviera muy convencido, esto lo habrá oído muchas veces y ha tenido que dejar cierto pose en su alma. Era el hombre ideal primero en el mito, del que se apoderaron las tinieblas, pero que conservó la luz hasta tornar a su propia perfección. Bastaba trasladar esto a la historia y tendríamos explicados muchos puntos de la doctrina agustiniana.

A su vez, la formación platónica y plotiniana, le habla de lo sensible y de la "caída", "siendo lo sensible, en un sentido, constituído por la caida, y en otro, sirviendo para cumplirla" ${ }^{25}$. Como además la finalidad propuesta por esta filosofía era la unión y contemplación del Uno, que en su cristianización identificará con Dios Agustín, éste confiere una gran importancia a la caída y a lo sensible, de lo que tendrá que purificarse para lograr su objetivo. El P. de Lubac había advertido ya que no puede olvidarse la "caída" y la "expulsión" de las esferas superiores, cuando se trate de penetrar en la inteligencia del pecado original y la expulsión del paraíso en la antigüedad cristiana ${ }^{26}$. A esto habría que añadir todavía, para discernir bien la terminología agustiniana y su alcance, la teoría de la participción aplicada al pecado original y a sus efectos, en especial a la concupiscencia.

Estos elementos se completan y Agustín superará el materialismo maniqueo con el espiritualismo neoplatónico, fijándose como finalidad la unión y contemplación de Dios, arrancando del primer hombre como ideal con todas sus prerrogativas ${ }^{27}$. Una vez más lo teórico e ideal del neoplatonismo vendría hecho historia en los primeros padres y permitiría a Agustín una explicación relativamente fácil. Palpando además por propia experiencia que el objetivo que se había fijado era imposible para sus solas fuerzas por causas que irá investigando, recurrirá a explicarlo con el pecado original, buscando luego la gracia para rehacer lo deshecho.

25 J. Trouillard, La puruficatiom plotinienne, Paris 1955, p. 29.

26 De Lubac, H., Surnaturel, Paris 1946, 380-381; cfr. Tresmontant, C., La métaphysique du christianisme et la naissance de la philosophie chrétienne, Paris 1961, 249-457.

27 FUMAGALII, J. M., La dottrina della "Justitia hominis negli scritti antipelagiani di S. Agostino, Romae 1967; Slomkowski, A., L'état primitif de l'homme dans la tradition de l'Eglise avant saint Augustin, Paris 1928. 
IDEAL E IMPOSIBILIDAD: LAS "SORDES ANIMAE".

El neoplatonismo le había abierto una ventana a la esperanza, pero ya en Milán habría sufrido la desilusión y el desengaño en los llamados éxtasis frustrados o intentos de éxtasis ${ }^{28}$. Se habla en las Confesiones de la visión de lo invisible e incorpóreo, pero en la que no pudo permanecer, tornando a las cosas deleznables y pasajeras, trayendo una memoria de Dios, de aquello que había visto in ictu trepidantis aspectus ${ }^{29}$. Esta necesidad y esta experiencia le implicó en una búsqueda más decidida para lograr la finalidad que se había manifestado como la obra más auténtica del hombre en el mundo, aunque luego tenga que renunciar ante la dificultad y la imposibilidad, reduciéndose su acción a la purificación.

Fijo en la finalidad de la contemplación, examina el porqué de la imposibilidad y considera la necesidad de la purificación. Pero ante la purificación de la que le habla en todos los tonos una filosofía plotiniana, Agustín desarrolla el análisis del objeto de esa purificación. Tal vez en este tema radique uno de los fundamentos esenciales de toda la doctrina posterior que ha cristianizado con palabras de la Escritura y ha identificado con los efectos del pecado. Si seguimos los pasos de Agustín, descubriremos su avance ascensional, su acercamiento a una expresión más bíblica del pensamiento y una profundización en el problema del pecado. Para ello nos detendremos de modo especial en los Diálogos con breves incursiones en las obras posteriores hasta el 396-397. El mismo dirá a Julián de Eclana que no ha inventado nada y que "ya desde el principio de su conversión siempre mantuvo lo que ahora mantiene".

"Exstant -continúa- libri quos adhuc laicus recentissima mea conversione conscripsi, etsi nondum sicut postea sacris Litteris eruditus, tamen nihil de hac re jam tunc sentiens, ut ubi disputandi ratio poposcerat dicens, nisi quod antiquitus discit et docet omnis Ecclesia: in has videlicet magnas manifestasque miserias, in quibus homo vanitati similis factus est, ut dies ejus sicut umbra praetereant (Ps 143, 4), et sit universa vanitas omnis homo vivens ( $\left.P_{s} 38,6\right)$, merito peccati originalis genus humanum fuisse collapsum; unde non liberat nisi qui dixit, Veritas liberabit vos (Jo 8, 32); et, Ego sum veritas (Id. 14, 6); et, Si vos Filius liberaverit, tunc vere liberi estis (Id. 8, 36). Non enim a vanitate nisi veritas liberat); sed secundum gratiam, non secundum debitum; per misericordiam, non

28 Cfr. Confess. VII, 10, 16, PL. 32, 742; 17, 23, col. 744-745; 20, 26, col. 746-747.

29 Courcelle, P., Recherches sur les Confessions de saint Augustin, Paris $1950,157-167$. 
per meritum. Sicut enim judicii fui ut vanitati subjiceremur; ita misericordiae est ut veritate liberemur, et ipsa bona merita nostra nonnisi Dei dona esse fateamur" 30.

Las palabras de Agustín no pueden ser más claras y la tradición a que se refiere la expresa con los términos tradicionales y con aquellos que en realidad se hallan en sus primeros escritos y en los anteriores a la controversia pelagiana: Vanidad en la que está inmerso el hombre y verdad liberadora, que se identificará con el Cristo. Sin embargo, en torno al texto se levantó una gran polémica y en ella intervinieron autores de una y otra tendencia ${ }^{31}$. El P. Casamassa entró de lleno en el argumento y concluiría diciendo que "el examen directo de las obras agustinianas, anteriores al 396-397, confirma luminosamente las categóricas afirmaciones del Contra Iulianum VI, 12 39" ${ }^{82}$.

Se pone la máxima insistencia en el cuerpo y en la mortalidad, como consecuencias del pecado en las primeras obras y luego en la concupiscencia, en la difficultas y en la ignorancia, que sin embargo, dicen casi siempre relación a lo corporal, como tendremos ocasión de señalar. La conclusión que se nos impone está conforme plenamente con las palabras de Agustín: El ha comenzado por afirmar, ya desde su conversión, la existencia de unas consecuencias de un pecado, enseñando "quod antiquitus discit et docet omnis Ecclesia", pero recuerda que no lo ha hecho con términos bíblicos, "etsi nondum sicut postea sacris Litteris eruditus". Es decir, en general se ha atenido a la afirmación de unos efectos, que le resolvían los problemas planteados también por la filosofía, pero, como en otros terrenos, ha ido avanzando en la comprensión, en la explicación y en terminología escriturística. La Escritura vendría luego, como le ha sucedido con frecuencia, y su avance en ella, nos lo deja bien manifiesto en las mismas Retractationes ${ }^{\mathbf{3}}$.

30 Contra Julian. VI, 12, 39, PL. 44, 843.

31 Principalmene fue E. Buonaiuti quien suscitó la cuestión, en su opúsculo La genesi della dottrina agostiniana in torno al peccato originale, Romia 1916, con sólo 23 p. En plan agresivo le respondía CoNCETTI, N., Esame della Genesi della dottrina agostiniana in torno al peccato originale di Ernesto Buonaiuti della Università di Roma, Fermo 1922, y con más profundidad y copia de argumentos y de textos el P. Antonio Casamassa, en el artículo que citamos a continuación.

32 Casamassa, C., Il pensiero di sant'Agostino nel 396-39\%. I "Tractatores divinorum eloquiorum" di Retract. I, 23, 1 e l'Ambrosiastro, Roma 1919, publicado ahora en Scritti patristici, I. (Lateranum n. s., an. XXI, n. 1-4, Roma 1955, 41-66), 48.

${ }^{33}$ Cfr. Bardy, G., Les Revisions. (Oeuvres de saint Augustin, 12), Paris $1950,83-105$. 
Siendo esto así, queremos iniciar el estudio por las primeras obras de Agustín deteniéndonos un poco en cada uno de los pasajes de las mismas en que aparecen esas manchas del alma - sordes animae-, que le privan del dominio del cuerpo y a veces la someten a él -consuetudo corporum - y no le permiten la visión de lo superior. Así comprendemos la secuencia progresiva del pensamiento agustiniano en este punto y quizá seremos al final más capaces de comprender la controversia pelagiana, en la que Agustín se cansa de repetir que cuanto está defendiendo no es nada nuevo para él, pues defiende la propia experiencia, es decir la imposibilidad de lograr la visión de Dios y la unión al mismo por las propias fuerzas, explicándolo por un pecado. Había comenzado con la filosofía y ha ido enriqueciéndose con la meditación y la Escritura.

EXAMEN DE LOS PRIMEROS ESCRITOS.

En la invitación a Romaniano a entrar en el puerto de la filosofía a través del Contra Academicos aparece en alguna ocasión la necesidad de la purificación para intuir la sabiduría, por ejemplo cuando le expone la fábula de ia filocalia y de la filosofía ${ }^{34}$. El mismo confiesa que no hace más que purificarse de sus faltas y vanas opiniones para llegar a la sabiduría y entrar limpiamente en la filosofía, llegando a la verdad ${ }^{35}$. Toda su tarea en Casiciaco se ha reducido precisamente a este primer intento expresado desde el principio. Trata de purificarse de la falsedad, quitando de ese modo "impedimenta inveniendae veritatis", porque ama la verdad extremamente y no la hallará si no entra totalmente en la filosofía ${ }^{36}$.

Ya la finai de la obra, tras la enumeración de las contenciones de los filósofos, de platónicos y aristotélicos, se refugia en la filosofía verdadera, no en la que detestan las Escrituras, sino en la auténtica, y escribe, recurriendo a la humildad de Dios que se abaja para curar y purificar las mandas y la soberbia del alma humana:

34 C. Acad. II, 3, 7, PL. 32, 922: "Ergo ille, si veram pulchritudinem cujus falsae amator est, sanatis renudatisque paululum oculis posset intueri, quanta voluptate philosophiae gremio se involverit?".

35 Id. II, 3, 9, PL. 32, 923: "Ego enim nunc aliud nihil ago, quam me ipse purgo a vanis perniciosisque opinionibus. Itaque non dubito melius mihi esse, quam tibi".

36 Id. II, 3, 8, PL. 32, 923: “...nam ipsum verum non videbis, nisi in philosophiam totus intraveris". 
"Non enim est ista hujus mundi philosophia, quam sacra nostra meritissime detestantur, sed alterius intelligibilis; cui animas multiformibus erroris tenebris caecatas, et altissimis a corpore sordibus oblitas, numquam ista ratio subtilissima revocaret, nisi summus Deus populari quadam clementia divini intellectus auctoritatem usque ad ipsum corpus humanum; declinaret, atque submitteret; cujus non solum praeceptis sed etiam factis excitatae animae redire in semetipsas, et respicere patriam, etiam sine disputationum concertatione potuissent" 37.

Tenemos ya señaladas dos cosas que prohiben al alma la vuelta a la primera que promete la filosofía: de una parte, las multiformes tinieblas del error, y de otra, las profundísimas manchas procedentes del cuerpo. La claridad no puede ser mayor, pero nos descubre a nosotros un mundo para el pensamiento que se irá haciendo luz en torno al pecado, y por tanto, también en torno a la encarnación y a la redención, que ahora se le aparece de ese modo, como lo recordará también en las Confesiones ${ }^{38}$. La filosofía lo dejaba ahí, aunque necesite explicación. Ahora señala hechos solamente y luego les hallará la solución.

En el De beata vita estos pensamientos se amplían hacia un nuevo campo de investigación, ya que ahora será la sabiduría la que dará la felicidad, pero a ella hay muchas cosas que se oponen. $Y$ la primera gran dificultad para llegar al puerto de la sabiduría y de la felicidad es el "superbum studium inanis gloriae", que es el monte que se alza ante quien pretende entrar en el puerto ${ }^{89}$. Como el alimento del alma es "rerum intellectus et scientia" ${ }^{40}$, resulta que el alma de los imperitos está llena de vicios y nequicia, y esto viene llamado por Agustín con el nombre de "quaedam sterilitas et quasi fames animorum" ${ }^{41}$. Esas son "enfermedades" y la "nequicia es madre de todos los vicios". Agustín va en busca del sapiens y éste será el auténticamente feliz. Por eso se ha entretenido en ver cómo era el ánimo de los imperitos, que no han quitado todavía de él a través de la disciplina y de la doctrina la nequicia y el error. Todos, por consiguiente, de principio vienen con esos vicios primeros, pero unos se han purificado y otros continúan en sus enfermedades y en su esterilidad y en su hambre. Les falta el conocimiento y la ciencia de las cosas.

3.7 Id. III, 19, 42, PL. 32, 956-957.

38 Confess. VII, 18, 24, PL. 32, 745-746; 20, 26, col. 746-747; 21, 27, cols. 747-748.

39 De beata vita 1, 3, PL. 32, 960-961.

40 Id. 2, 8, PL. 32, 963.

41 Ib., col. 964 . 
El niño, es decir Adeodato entonces dijo que "aquel tiene a Dios, que no tiene espíritu inmundo. Y la madre, Mónica, aprobó sobre todo esto" ${ }^{42}$. De una manera o de otra consintieron en esta profesión de fe común, refiriéndose a la carencia de espíritu inmundo. Y se apresta luego a considerar y definir el sentido de espíritu inmundo en un doble aspecto, sin duda en parte tomado de los misterios cristianos. Lo explica en los siguientes términos, sumamente importantes, porque nos acercan por pasos al misterio cristiano, aunque bastante imprecisamente:

\footnotetext{
"Tertium vero illud paulo diligentius considerandum est, propterea quod ritu castissimorum sacrorum spiritus inmundus, quantuml intelligo, duobus modis appellari solet: vel ille qui extrinsecus invadit animam, sensusque conturbat, et quemdam hominibus infert furorem, cui excludendo qui praesunt, manum imponere vel exorcizare dicuntur, hoc est divina eum adjurando expelleret: aliter autem dicitur spiritus inmundus, omnis omnino anima inmunda; quod nihil es aliud quam vitiis et erroribus inquinata" 48.
}

Agustín se debate en un cristianismo de primera mano, sin filosofías ni teologías, cuyos términos no comprende bien, pero que trata de explicar con sus concepciones filosóficas. Los principales interlocutores de este diálogo eran Mónica y Adeodato y de ahí que se apele con frecuencia a los misterios aristianos, sencillamente referidos. El espíritu inmundo es el alma manchada de vicios y de errores y Mónica añadirá que "quien por los vicios y pecados se aleja de Dios, no sólo no sea feliz, sino que no viva con un Dios propicio" ${ }^{44}$.

Una vez más tenemos el hecho de los errores y de los vicios, sin especificarse la fuente, aunque la referencia a los misterios cristianos quiere indicar algo. El proceso continúa; Agustín se explica en términos filosóficos y cuando topa con el alma encerrada en la prisión del cuerpo y con algo que se le asemeja en los misterios cristianos trata de poner en relación aquéllos con éstos.

Y torna a la idea que ya había expuesto antes, a saber que la stultitia es egestas, oponiéndose a la sabiduría. Con aquélla no puede ser feliz, convirtiéndose la stultitia y la egestas ${ }^{45}$, y siendo el stultus también

42 Ib. 2, 12, PL. 32, 966: "Puer autem ille minimus omnium: Is habet Deum, ait, qui spiritum immundum non habet. Mater omnia, sed hoc maxime approbavit. Navigius tacebat". Cfr. también 3, 17, col. 968.

43 Id. 3,18 , PL. 32. 968-969.

44 Id. 3, 21, PL. 32, 970: "quisquis vitiis atque peccatis a Deo se alienat, non modo beatus non sit, sed ne Deo quidem vivat propitio".

$45 I d, 4,29$, PL. 32, 973. 
miser ${ }^{46}$. La mayor falta será, en consecuencia, la estulticia, que es defecto para el mismo ser humano que aspira con todas sus fuerzas a la sabiduría, llegando a la felicidad si logra la sapiencia. Los fundamentos están sentados: la sabiduría acerca a la felicidad y la estulticia aleja de ella. El hombre tiene que huir de la ignorancia, purificándose de sus errores, para entrar en la región de la sabiduría. Pero gde dónde le viene al ánimo la estulticia? Agustín no responde todavía a la pregunta. Consta el hecho y lo deja sin explicación.

Feliz será quien tenga la sabiduría y la sabiduría verdadera es la sabiduría del Padre, el Hijo de Dios ${ }^{47}$. La conclusión del diálogo sobre la vida feliz da el proceso de la estulticia y de la sapiencia, destilando ques es feliz quien no necesita nada, y que éste es el sabio, definiendo qué es la sabiduría, que nosotros traduciríamos también por equilibrio ${ }^{48}$. La sabiduría se propone como medio de huir a los placeres y a los temores, a los vicios que manchan y afean el alma. Vemos a través de las cuatro palabras que jalonan el texto algo que tiene repercusión en las virtudes cardinales, tales como las entiende el Santo, siguiendo a Cicerón: sordibus, timoribus, moerore, cupiditate, que son los cuatro principales elementos pasionales, de que nos habla con frecuencia en sus obras, como contrapuestos a las virtudes.

El tema de la estulticia y por tanto de la ignorancia es abordado desde la entrada misma dei De ordine. Se constata ya como una realidad y se parte también del sapiens a que se aspira. El hombre no atribuye a la providencia cuanto sucede en el mundo por el hecho de ignorarse a sí mismo y se ignora a sí mismo por estar implicado entre las cosas por

$46 I d .4,28$, PL. 32, 973.

47 Id. 4, 34, PL. 32, 975-976.

48 Id. 4, 33, PL. 32, 975: "Dixeramus enim in exordio hodiernae disputationis nostrae. quod si inveniremus nihil aliud esse miseriam quam egestatem, eum beatum esse fateremur. qui non egeret. Est autem inventum: ergo beatum esse nihil est aliud quam non egere, hoc est esse sapientem. Si autem quaeritis quid sit sapientia (nam et ipsam ratio, quantum in praesentia potuit, evolvit atque eruit); nihil est aliud quam modus animi. hoc est, ouo sese animus librat, ut neque infra ouam plenum est coarctetur. Excurrit autem in luxurias, dominationes, superbias, coeteraque id genus, quibus immoderatorum miserorumaue animi sibi laetitias ataue potentias comparari putant. Coarctatur sordibus, timoribus, moerore, cuniditate, atrue aliis, ouafcumoue sunt. ouihus homines miseros etiam miseri confitentur. Cum vero sapientiam contemplatur inventam, cumaue, ut hujus pueri verbo utar, ad ipsam se tenet, nec se ad simulacrorum fallaciam, ouorum pondus amplexus a Deo suo cadere atque demergi solet, ulla commotus inanitate converti; nihil immoderationis, et ideo nihil egestatis, nihil igitur miseriae pertimescit. Habet ergo modum suum, id est sapientiam quidquid beatus est". 
los sentidos. Se le impone, pues, un apartarse de los sentidos y entrar en sí mismo, soledad y medicina de las disciplinas liberales ${ }^{49}$. En las Retract. I, 3, 2 (PL. 32, 588) dirá que le desplace no haber añadido corporis, cuando habla aquí de sensus. Era esa su intención, al menos cuando revisa su obra. Corregirá también o pondrá en tela de juicio la gran atribución y el papel que ha comisionado a las disciplinas liberales, como si ellas pudieran sanarlo todo. El ánimo debe comenzar por volver a sí mismo para contemplar la belleza del universo ${ }^{50}$. La unidad la pide el universo o universitas que de uno se llama así, mas la entrega a la multiplicidad cierra al hombre el camino de la contemplación. Tomando la imagen del geómetra y del centro en torno a la unidad describe la multiplicidad de este modo:

"hinc vero in.quamlibet partem si egredi velis, eo amittuntur omnia, quo in plurima pergitur: sic animus a seipso fusus inmensitate quadam diverberatur, et vera mendicitate conteritur, cum eum natura sua cogit ubique unum quaerere, et multitudo inveniri non sini" 51 .

En busca de la belleza, por la igualdad y la unidad, da en la multiplicidad que se opone a ello y no le permite el ingreso fácil y libre. Es, en el fondo, la huída de sí mismo, la ignorancia, el estar preso de los sentidos lo que le niega la visión de la belleza.

La presentación a Zenobio no puede ser más sugestiva y el programa que pretende llevar a cabo más espléndido. El problema es siempre el mismo: la purificación para la visión clara. En ella aparece la búsqueda de las causas del error y se revela la inmoderación de la libidine y las manchas, las perniciosas cupididades y las falsas voluptuosidades, de todo lo cual es necesario despojarse ${ }^{52}$. Antes de ser entregados los "semina

49 De ord. I, 1, 3, PL. 32, 979: "Cujus erroris maxima causa est, quod sibi ipse est incognitus. Qui tamen ut se noscat, magna opus habet consuetudine recedendi a sensibus, et animum in seipsum colligendi atque in seipso retinendi. Quod ii tantum assequuntur, qui plagas quasdam opinionum, quas vitae quotidianae cursus infligit, aut solitudine inurunt, aut liberalibus medicant disciplinis".

50 Id. 2, 3, PL. 32, 979 .

51 Ibid., PL. 52, 980.

52 Id. I, 2, 4, PL. 32, 980: "Sed et haec quae dixi qualia sint, et quae causa exstet erroris animarum, quoquo modo et in unum congruant atque perfecta sint, cuncta, et tamen peccata fugienda sint, assequeris profecto, mi Zenobi. Sic enim mihi notum est ingenium tuum, et pulchritudinis omnimodae amator animus, sine libidinis immoderatione atque sordibus. Quod signum in te futurae sapientiae perniciosis cupiditatibus divino jure praescribit, ne tuam causam deseras falsis voluptatibus illectus; qua praevaricatione nihil turpius et periculosius inveniri potest. Assequeris ergo ista, mihi crede, cum eruditioni operam dederis, qua purgatur et excolitur animus, nullo modo ante idoneus, cui divina semina conmitantur". 
divina" al alma, ésta necesita purificarse y cultivar el espíritu por medio de la erudición. Conste que no se hable todavía de otra cosa, aunque más tarde diga, mucho más tarde, que ha concedido demasiado a las disciplinas y a la erudición. Eran medios para la purificación y el cultivo del ánimo. Se trata de no ceder a la inmoderación de la líbidine y de las "sordes" y de resistir a las "cupiditates", tratando de oprimirlas ${ }^{58}$. El fin de todo ello es convertirse a Dios, desde la región en que se halla sometido a lo pernicioso y erróneo, a lo feo y a lo tenebroso:

"A quibus enim rebus -escribe- putas nos orare ut convertamur ad Deum, ejusque faciem videamus, nisi a quodam coeno corporis et sordibus, et item a tenebris quibus nos error involvit? Aut quid est aliud converti, nisi $a b$ inmoderatione vitiorum, virtute ac temperantia in sese attolli?" 54.

$\mathrm{La}$ "scabies voluptatum aerumnosarum" $" 55$ es necesario curarla, teniendo como medicamentos la erudición y las disciplinas liberales. Una y otra vez se recurre a los mismos términos y la curación final se comisiona a Cristo, que providencialmente ha mandado Dios para sanar de esos males :

"Nonne vos movet quibus vitiorum molibus atque imperitiae tenebris premimur et cooperiamur?... Miseri, nescitis ubi sumus? Demersos quidem. esse animos omnium stultorum indoctorumque commune est, sed non uno, atque eodem modo demersis, opem sapientis et manum porrigit. Alii sunt, credite, alii sunt qui sursum vocantur, alii qui in profunda laxantur. Satis mihi sint vulnera mea, quae ut sanentur, pene quotidianis fletibus Deum rogans, indigniorem tamen esse me qui tam cito saner quam volo, saepe memetipso convinco" 56 .

Y entonces el alma del sapiens, purificada por las virtudes, deja a un lado las "sordes quaedam et exuviae" ${ }^{57}$.

Examinando los grados de las disciplinas y su medida para la purificación se centra nuevamente sobre la estulticia que es "pestis animae" ${ }^{8}$,

53 Id. I, 4, 10, PL. 32, 983: “Ergo aggrediamur, Licenti, freti pietate cultores, et vestigiis nostris ignem perniciosum fumosarum cupiditatum opprimamus".

54 Id., I, 8, 23, PL. 32, 988.

55 Id. I, 8, 24, PL. 32, 988.

56 Id. I. 10, 29, PL. 32, 991.

ธ7 Id. II, 2, 6, PL. 32, 996.

58 Id. II. 3, 8, PL. 32, 998. 
y con cuya definición no se acierta fácilmente ${ }^{8}$. Una cosa puede decirse, asegura, y es que hay que evitarla como las tinieblas ${ }^{60}$.

En este discurso aparecen ya los diversos males y las acciones no buenas cometidas en el mundo y se pregunta si se ralizan con orden y si van regidas por el mismo, entrando en otras cuestiones para venir a desembocar en la autoridad como purificación ${ }^{61}$. Tras un examen de todas las artes liberales y del método de ese estudio y de cómo puede avanzarse de corporalibus ad incorporalia, llega al fin. $\mathrm{Y}$ hace constar que, huyendo de lo perecedero y tornadizo, se alza el entendimiento a lo divino y a la comprensión - "contemplanda", "intelligenda" y "retinenda"- de las diferentes cuestiones filosóficas, pero errará en el conocimiento "si servus adhuc cupiditatum, et inhians rebus pereuntibus" "2. Para lograr la visión y la contemplación del plan divino hay que apartarse de todas las fugacidades y emerger de la mancha del cuerpo con la edad o con una admirable templanza ${ }^{68}$.

Todo esto era comprensibilísimo, dado el convencimiento, nacido de los contactos en que Agustín estaba sumido, de que a Dios solamente lo ven los puros. El concepto es plenamente plotiniano y su fuente sería hallada. Esta purificación será clave en ia teología agustiniana.

La aplicación práctica de cuanto nos ha revelado en los Diálogos nos la ofrece Agustín en los Soliloquios. Ya en la plegaria inicial anuncia: "Deus qui nisi mundos verum scire noluisti" ${ }^{64}$. Y poco más adelante:

"Deus, quem nemio amittit nisi deceptus; quem nemo quaerit, nisi admonitus; quem nemo invenit, nisi purgatus" 65 .

Y como además El era el "pater pugnoris quo admonemur redire ad te" ${ }^{66}$, resuita que es El mismo quien llama para que se le busque y a quien se le halla, purificado. A esa purificación dedicará todo el primer

59 Id. II, 3, 9, PL. 32, 998-999.

60 Id. II, 3, 10, PL. 32, 999: “...ut enim oculis tenebras vitamus eo ipso quo nolumus non videre: sic quisquis volet vitare stultitiam, non eam conetur intelligere; sed ea quae posunt intelligi, per hanc se non intelligere doleat; eamque sibi esse praesentem, non quo ipsam magis intelligit, sed quo alia minus intelligit, sentiat".

61 Id. II, 9, 27, PL. 32, 1.008.

62 Id. II, 16, 44, PL. 32, 1.015.

63 Id. II, 17, 45, PL. 32, 1.015-1.016: “...tibi tamen cujus ingenium quotidie mihi novum est, et cujus animum vel aetate vel adnvirabili temperantia remotissimum ab omnibus nugis, et magna labe corporis emergentem, in se multum surrexisse cognosco, tam erunt facilia quam difficilia tardissimis miserrimeque viventibus".

64 Solil. I, 1, 2, PL. 32, 870.

65 Id. I, 1, 3, PL. 32, 870 .

66 Id. I, 1, 2, PL. 32, 870. 
libro en un examen de conciencia, para llegar al conocimiento casi matemático de Dios. Para ver a Dios se precisan ojos sanos y

"oculi sani mens est ab omni labe corporis pura, id est, a cupiditatibus rerum mortalium jam remota atque purgata: quod ei nihil aliud praestat quam fides primo. Quod enim adhuc ei demonstrari non potest vitiis inquinatae atque aegrotanti, quia videre nequit nisi sana, si non credat aliter se non esse visuram, non dat operamı suae sanitati" ${ }^{67}$.

Apelará luego, en breve, a las tres virtudes, fe, esperanza y caridad, para escapar a las molestias del cuerpo ${ }^{68}$. Lo demás en esta obra es ya conocido.

En el De inmortalitate animae vuelve en alguna ocasión sobre el "animus stultus" y su "aversio a ratione", explicando qué significa eso ${ }^{69}$. El error en la apreciación de la naturaleza del ánimo lo achaca a la falta de purificación. Así escribe :

"Nisi forte vitam temperationem aliquam corporis, ut nonnulli opinati sunt, debemus credere. Quibus profecto numquam hoc visum esset, si ea quae vera sunt et incommutabilia permanent, eodem animo a corporum consuetudine alienato atque purgato videre valuissent" 70.

Explícitamente se nos hace referencia a algo que se ampliará luego y que estaba ya implícito en todo lo precedente, es decir a la "corporum consuetudo".

Los Diálogos de Casiciaco nos han referido hechos: la ignorancia, el error, las tinieblas, la estulticia, el cuerpo y sus obstáculos, que arrastran hacia placeres y cupididades - libidines, voluptates, cupiditates-. Todo ello constituyen las "sordes animae" que, dada la insistencia que Agustín pone en el cuerpo, proceden sin duda alguna de lo corporal, de lo sensible y por eso "fugiendum est a corpore" "1 será la palabra de orden en estos primeros escritos, máxime cuando se trata de la veracidad de los sentidos. Ofrece esto un interés muy especial, porque son hechos de experiencia, conscienciados por la filosofía, y serán fondo común de la doctrina posterior.

Sin embargo, anotamos todavía más: Agustín no ha afirmado nada

67 Id. I, 6, 12, PL. 32, 875-876.

68 Id. I, 7, 14, PL. 32, 876-877.

69 De inmort. animi. 7, 12, PL. 32, 1.027; 8, 13, PL. 32, 1.027-1.028.

70 Id. 10, 17, PL. 32, 1.029 .

71 Cfr. Epist. 3, 4, PL. 33, 65; Solil. I, 3, 8, PL. 32, 873; 14, 24, PL. 32, 882 ; De quant, anim. 28, 55, PL. 32, 1.066; De div. quaest. 83, q. 9, PL. 40, 13. 
aún sobre la existencia o menos de un pecado. Bien es cierto que el origen del mal le había preocupado ya durante su estancia en el maniqueismo y se lo había venido planteando muy seriamente. En su alma seguía en suspenso la respuesta a la pregunta: ¿De dónde proceden esas "sordes animae"? En Casiciaco, a juzgar por los escritos no tiene todavía una respuesta, aunque no sea más que como afirmación. Había sólo brevísimas referencias a la creación y a la caída. Tendremos que esperar hasta después del bautismo para hallar las primeras afirmaciones del pecado o del peccatum antiquum. Esto obligaría a examinar otro punto, su relación con Ambrosio durante este tiempo ${ }^{72}$.

$\mathrm{Si}$ quisiéramos continuar con los diferentes pasajes, anteriores al año 396-397, que hablan del pecado o del pecado antiguo, sin concepto propio de pecado, toparíamos con las expresiones analizadas. Ha habido en todo ello muy poca evolución. La insistencia prosigue puesta sobre el cuerpo, la mortalidad, la corruptibilidad y la dificultad que ocasiones -recuérdense los famosos pasajes del De musica y los discutidos dei De libero arbitrio-. Con ello obliga a una atención mayor a la "consuetudo corporum" y a una cierta "necessitas", que recordará todavía seriamente en las Confesiones, como experiencia muy personal.

Agustín decía en una síntesis que recoge un poco el pensamiento de los pasajes citados con referencia además al "peccatum antiquum":

"Sed inter omnio quae in hac vita possidentur, corpus homini gravissimum vinculum est, justissimis Dei legibus, propter antiquum peccatum, quo nihil ad praedicandum notius, nihil ad intelligendum secretius. Hoc ergo vinculum ne concutiatur atque vexetur, laboris et doloris; ne aufeergo atque perimatur, mortis terrore animam quatit. Amat enim illud vi consuetudinis, non intelligens, si eo bene atque scienter utatur, resurrectionem reformationemque ejus ope ac lege divina sine ulla molestia juri suo subditam fore: sed cum se hoc amore tota in Deum converterit, hos cognitis mortem non modo contemnet, verum etiam desiderabit" 73.

Hay algunos hechos dignos de nota en este texto. Agustín continúa con el sentido platónico del cuerpo como vínculo. $\mathrm{Y}$ a su vez achaca esto al pecado antiguo, más conocido que el cual no hay nada para predicar

72 Nos llevaría muy lejos este examen y está en preparación. De cuanto hemos podido investigar hasta el presente aparece claro que los términos usados por Agustín en esas obras y que puede decir relación a ese primer pecado o a sus efectos son idénticos a los empleados por Ambrosio en los sermones que posible o probablemente Agustín oyó durante su preparación al bautismo.

78 De mor. eccl. cath. I, 22, 40, PL. 32, 1.328. 
ni más secreto para entender. El no había predicado todavía, pero sin duda había oído hablar de ello en un platonismo cristianizado como era el de Ambrosio. Referencia a los efectos corporales nos la hace en otros pasajes ${ }^{74}$. Y así por ejemplo dice también:

"Mag.-Mirare potius quod facere aliquid in anima corpus potest. Hoc enim fortasse non posset, si non peccato primo corpus illud nulla molestia et summa feliciate animabat et gubernabat, in deterius commutatum, et corruptioni subjaceret et morti..." 75 .

\section{Y agrega en otra ocasión:}

Non enim sicut peccatum in ejus potestate est, ita etiam poena peccati. Magna quippe res est ipsa anima, nec ad opprimendos lascivos motus suos idonea sibi remanet. Valentior enim peccat, et post peccatum divina lege facta imbecillior, minus potens est auferre quod fecit" 76.

El probiema de la acción del alma en el cuerpo o viceversa o de cómo puede actuar el cuerpo en el alma tiene una gran transcendencia en este libro VI De musica y Agustín apelará a la dificultad de movimientos que tiene el alma en el cuerpo por causa de ese mismo no dejarse dominar. Creerá que eso se debe al "primer pecado". Hay otra serie de textos, sobre todo del De vera religione ${ }^{77}$ y dei De Gen. contra manichaeos ${ }^{78}$ que insisten en lo mismo, acentuando algún otro aspecto. $\mathrm{La}$ insistencia va sobre la "poena peccati", que en general viene identificada con la dificultad para regir el cuerpo y con la ignorancia. $\mathrm{Y}$ así leemos en otra parte:

"In corporibus autem inferioribus atque mortalibus post anima ordinata, regit corpus suum, non omnimodo pro arbitrio, sed sicut leges universitatis sinunt. Nec ideo talis anima inferior est corpore coelesti, cui corpori etiam corpora terrena subjecta sunt. Pannosa enim vestis damnati servi multo est inferior veste bene mariti, et in honore magno apud Dominum constituti; sed ipse servus melior est qualibet veste pretiosa, quia homo est" 79 .

74 Cfr. Id. I, 19, 25, PL. 32, 1.326 y en los textos de las notas siguientes.

75 De mus. VI, 4, 7, PL. 32, 1.166.

76 Id. VI, 5, 14, PL. 32, 1.170.

77 De vera relig. 12, 25, PL. 34, 132-133; 15, 29, PL. 34, 134; 20, 38, PL. 34, $138 ; 44,82$, PL. $34159 ; 45,83$, PL. $34,160$.

78 De Gen. contra manich. I, 13, 19, PL. 34, 182; 18, 29, PL. 34, 187; II, 7 , 8, PL. 34, 200; 19, 29, PL. 34, 210-211; 21, 31-32; PL. 34, 212-213; 25, 38, PL. 34, 216; 27, 41, PL. 34, 218; 29, 29, 43, PL. 34, 219-220.

79 De libero arb. III, 11, 34, PL. 32, 1.287-1.288; cfr. también 10, 31, PL. 32, 1.286 ; 18, 51-52, PL. 32, 1.295-1.296; 19, 53-54, PL. 32, 1.296-1.297; 20, 65, PL. 32, 1.297. 
Agustín tenía todavía un problema sin resolver, que intuía, pero ai que no había dado forma definitiva: era el origen de todo esto. Se ofrecían a su vista dos caminos: o el maniqueismo con doble principio, o la creación, que ya admitía y en cuya explicación invertirá mucho tiempo. Ante los hechos que ha constatado por experiencia y por la filosofía, busca en la Escritura un punto de apoyo y descubrirá la primera caída como la causa de todo ello, siendo entonces la voluntad del hombre la causa de la deficiencia, dependiendo de ella todas las demás. En el $D e$ libero arbitrio anunciaba, aún imprecisamente, la encarnación y la redención con un parangón a contrario entre la caída y la justicia del Verbo de Dios al redimir ${ }^{80}$. Engañada la mujer y por ella el varón toda la descendencia de éste viene castigada a las leyes de la muerte. Es la puesta en vista de los efectos que ha señalado en los textos y ia relación con la primera caída y el engaño. Tal como entendía entonces los efectos de aquel primer pecado, así entendería también la redención.

Todos estos son puntos de reflexión y meditación a través de los textos agustinianos ${ }^{81}$. Dada la trayectoria de su pensamiento y su ideal inicial que aparece muy claro en los Diálogos y en las obras de sus primeros escarceos intelectuales, no solamente en el fondo de su doctrina sobre el pecado original, sino en casi todas las demás, existe un presuplesto filosófico y un presupuesto existencial, vivido con hondura en su propia alma. Agustín fue madurando con los años su doctrina y ésta adquirió un tinte mucho más bíblico, al menos en su expresión. Bajo los textos de la Escritura juegan un papel primordial sus concepciones filo-

so Id. III, 10, 31, PL. 32, 1.286: "Atque Verbum Dei unicus Dei Filius, diabolum quem semper sub legibus suis habuit et habebit, homine indutus etiam homini subjugavit, nihil ei extorquens violento dominatu, sed superans eum lege justitiae; ut quoniam, femina decepta, et dejecto per feminam viro, omnem prolem primi hominis tanquam peccatricem legibus mortis, malitiosa quidem nocendi cupiditate, sed tamen jure aequissimo vindicabat, tamdiu potestas ejus valeret, donec interficeret justum, in quo nihil dignum morte posset ostendere, ron solum quia sine crimine occissus est, sed etiam quia sine libidine natus: cui subjugaverat ille quos ceperat, ut quidquid inde nasceretur, tanquam suae arboris fructus, prava quidem habendi cupiditate, sed tamen non iniquo possidendi jure retineret. Justissime itaque dimittere cogitur credentes in eum quem injustissime occidit, ut et quod temporaliter moriuntur, debitum exsolvant, et quod semper vivunt, in illo vivant, qui pro eis quod non debebat exsolvit". El subrayado de los textos latinos, citados tanto en el cuerpo del artículo como en las notas es nuestro.

81 Quizá las conclusiones debieran extraerse tras el análisis de todas las obras agustinianas y con un sentido más crítico. Sin embargo, nuestras primeras investigaciones y la misma bibliografía nos permiten adelantar las conclusiones, que continuaremos confirmando en estudios sucesivos sobre este tema tan interesante en el pensamiento agustiniano y en el de la tradición en general. 
sóficas iniciales, ya que cuando Agustín se encuentre con la Bibiia de verdad, ha madurado en su propio pensamiento.

Hemos querido indicar con estas palabras simplemente que Agustín tenía razón cuando decía a Julián de Eclana que había enseñado, "quod antiquitus dicit et docet omnis Ecclesia", pero con una anotación, "etsi nondum sicut postea sacris Litteris eruditus". Sus convicciones filosóficas hallaban una prueba y una confirmación en las Sagradas Letras, con el peligro de interpretar algunos textos según sus propias concepciones. Quizá de este quid pro quo Agustín no se diera cuenta precisa. No obstante, examinando la esencia del pecado original más tarde en el Santo y sobre todos sus efectos, ignorancia y concupiscencia, vemos con claridad que eso mismo lo había puesto de relieve por medio de la filosofía y merced a su construcción sobre la memoria $D e i$, que se trocará en la imagen de Dios.

Agustín no se ha inventado el pecado original, sino que ha descubierto en él el origen de la imposibilidad que tiene el hombre para cumplir su suprema aspiración que es la unión con Dios y la contemplación del mismo. Esto le explicaba los hechos constatados y le bastaba; de tal suerte que, al aparecer el pelagianismo, se vería obligado a defender una doctrina que había esclarecido con la experiencia y la filosofía y había explicado con la Escritura, hallándola también en ella. Sin embargo, todo esto ha sido ya estudiado en sus diferentes ramificaciones, como insinuamos al principio. Con estos presupuestos puede entenderse el porqué de algunas de sus interpretaciones a los textos escriturísticos, sin que entremos tampoco a examinar las sentencias sobre el origen del alma, que tanta trascendencia tendrá para la transmisión del pecado original.

\section{Conclusiones.}

Concluiríamos, pues diciendo:

I. Agustín, a través de su formación maniquea y neoplatónica -no entramos en el estoicismo- está llamado a admitir un hombre ideal, modelo, perfecto, que vería a Dios y podría adherirse a El.

2. Con la experiencia en la mano se percata de que existen unas dificultades que no le permiten esa finalidad. Son las "sordes animae", de las que tiene que purificarse, como le decía el neoplatonismo y su 
cristianismo le exigía ahora. $\mathrm{Y}$ esas "sordes animae" son el apego a lo sensible, la mortalidad, la conruptibilidad, la ignorancia, el error y la concupiscencia. Esta sería la línea tradicional, que la controversia le ha hecho profundizar, preguntándose por el origen. En esto radicará su aportación reflexiva.

3. Agustín habría traducido a la realidad concreta aquello que era el hombre ideal y vendría identificado, siguiendo la Escritura, en cierto modo con el primer Adán, a quien adornaría de todos aquellos bienes que el hombre ideal (filosófico o del mito maniqueo) gozaba. La privación de todo aquello vendría representado en las "sordes animae" y en la "consuetudo corporum" y estos efectos se deberían al pecado original.

4. El obispo de Hipona tenía razón cuando decía que en la controversia pelagiana enseñaba "quod antiquitus discit et docet omnis Ecclesia", pero "etsi nondum sicut postea sacris Litteris eruditus". Ha avanzado en terminología bíblica, pero a veces su exégesis es filosófica, es decir su contenido no es escriturístico siempre, sino filosófico, expresando en términos bíblicos lo que tenía adquirido por la experiencia y por la filosofía.

5. Por último, añadiríamos, aunque no haya sido éste nuestro tema, que en el De doctrina christiana, dando las reglas para una recta interpretación y progreso en el estudio de la Escritura dice que deben usarse todos los conocimientos necesarios, filológicos, geográficos, naturales, filosóficos, etc. Creeríamos que en la práctica también él ha tratado de emplear los conocimientos de su tiempo, aunque muchos de los consejos que ha prodigado en esta obra haya sido pura teoría para él y no los haya aplicado a su propia exégesis. Su relatividad estaría en esta misma regla y también la libertad para nuevas interpretaciones.

José Morán, O. S. A. 